\title{
Presacral tumors: diagnosis and treatment - a challenge for a surgeon
}

\author{
Łukasz Dziki, Marcin Włodarczyk, Aleksandra Sobolewska-Włodarczyk, Aleksander Saliński, \\ Magdalena Salińska, Marcin Tchórzewski, Michał Mik, Radzisław Trzciński, Adam Dziki
}

Department of General and Colorectal Surgery, Medical University of Lodz, Lodz, Poland

Submitted: 29 February 2016

Accepted: 27 June 2016

Arch Med Sci 2019; 15 (3): 722-729

DOI: 10.5114/aoms.2016.61441

Copyright $\odot 2016$ Termedia \& Banach

\begin{abstract}
Introduction: Presacral tumors are a rare group of heterogeneous lesions located in a potential space referred to as the retrorectal or presacral space. Lack of characteristic symptomatology and difficult anatomical localization make the diagnosis and management challenging for a surgeon. The aim of this study was to analyze cases of presacral tumors that underwent surgical treatment with regard to diagnostics, methods and outcomes.

Material and methods: The study enrolled patients who underwent surgical treatment at the Department of General and Colorectal Surgery, Medical University of Lodz. The data was analyzed for age, gender, clinical symptoms, type of diagnostic procedures conducted, histopathology results, type of treatment implemented, intra- and perioperative complications as well as early and long-term treatment outcomes.

Results: The study enrolled 29 patients who underwent surgical treatment for presacral tumors. Malignant tumors accounted for $34 \%$ of all cases $(n=10)$, and $80 \%$ of them occurred in men. Benign cases accounted for $66 \%$ of cases $(n=19)$, and they occurred predominantly in women (58\%). Malignant lesions were more common in men $(p<0.05)$. The average age of patients with benign tumors was lower than that of patients with malignant tumors $(p<0.05)$. The transsacral approach was used in $51 \%$ of patients, abdominal laparotomy in $41 \%$ and a combined approach in $7 \%$. Cure was achieved in $72 \%$ of patients, including $67 \%$ who were cured after their initial surgery. Conclusions: Presacral tumors are more common in men and more commonly are malignant tumors in this group. The success rate of surgical treatment is $72 \%$, and selection of the surgical approach does not affect the final treatment outcome.
\end{abstract}

Key words: presacral tumors, retrorectal tumors, surgical strategy, diagnosis.

\section{Introduction}

Presacral tumors are a rare group of heterogeneous lesions located in a potential space referred to as the retrorectal or presacral space. The estimated incidence of lesions in this region is $1 / 40,000$ admissions $[1,2]$. The classification of presacral tumors takes into account histology (benign or malignant tumors) and origin (congenital or acquired).

The presacral location is responsible for atypical symptoms with varying nature and intensity depending on the tumor size and coexisting in-

\author{
Corresponding author: \\ Prof. Adam Dziki MD, PhD \\ Department of General \\ and Colorectal Surgery \\ Medical University of Lodz \\ Pl. Hallera 1 \\ 90-647 Lodz, Poland \\ Phone: +48 535787848 \\ Fax: +48 426393076 \\ E-mail: adziki@wp.pl
}


flammation of surrounding tissues. The occurring symptoms are directly related to compression or infiltration of anatomical structures of the pelvis minor by the tumor and may manifest as lower back pain or rectal pain or resemble various neurological defects. However, often presacral tumor are asymptomatic; completely asymptomatic lesions occur in $26-50 \%$ of patients $[3,4]$. Lack of characteristic symptomatology and difficult anatomical localization make the diagnostic process difficult and often delays the ultimate diagnosis $[3,4]$.

The cornerstone of diagnosis of presacral tumors is the digital rectal examination (DRE), with sensitivity approaching 97\% [5]. Transrectal ultrasound imaging (TRUS), computed tomography (CT) and magnetic resonance imaging (MRI) provide assessment of their topography and relation to the other anatomical structures of the pelvis minor $[1,5,6]$.

Irrespective of histologic grade, the treatment of choice for presacral tumors involves surgical intervention from either an abdominal, transsacral, or mixed approach with or without resection of the cocygeal bone. Selection of the surgical technique depends on the size and level of the tumor location with respect to the S3 vertebra. Some histological types of presacral tumors require additional chemo- or radiotherapy, but such management is controversial due to high radioresistance of tumors of this region [3].

The aim of this study was to analyze cases of presacral tumors that underwent surgical treatment at the Department of General and Colorectal Surgery, Medical University of Lodz between 2003 and 2012 with regard to diagnostics, methods and outcomes of treatment.

\section{Material and methods}

The study enrolled patients who underwent surgical treatment due to presacral tumors at the Department of General and Colorectal Surgery, Medical University of Lodz from 2003 to 2012. The study data were retrospectively collected from medical records, surgical protocols, histopathology reports and a questionnaire completed by patients. The data were analyzed for age, gender, clinical symptoms, type of conducted diagnostics procedures, histopathology results, type of implemented treatment, intra- and perioperative complications as well as early and long-term treatment outcomes. Follow-up was evaluated based on data from outpatient clinics of surgery and a questionnaire completed by all patients who had undergone surgical treatment. In all patients the surgical approach for presacral tumors is established by appropriate imaging methods (TRUS, CT and MRI), which demonstrate the location, nature, and size of the lesion as well as the involvement of adjacent viscera, sacrum, or pel- vic sidewalls. The extent of surgery is determined by the character of the tumor, and in all cases the decision was made intraoperatively.

\section{Statistical analysis}

The collected data were analyzed using the Statistica 10.0 software package (StatSoft, Inc., United States). Student's t-test and Fisher's exact test were used to analyze variables specified on an interval scale. $P<0.05$ was considered statistically significant.

\section{Results}

The study enrolled 29 patients who underwent surgical treatment for presacral tumors. This group included 16 men (average age: 48 years) and 13 women (average age: 46 years). Surgical treatment for presacral tumors accounted for $0.19 \%$ of all surgical procedures performed in the analyzed period at the Department of General and Colorectal Surgery, Medical University of Lodz.

Malignant tumors accounted for $34 \%$ of all surgical cases $(n=10)$, and $80 \%$ of them occurred in men. Benign tumors accounted for $66 \%$ of cases $(n=19)$ and they occurred predominantly in women (58\%). Benign lesions were more common in women, while malignant lesions were more common in men $(p<0.05)$. The average age of patients with benign tumors was lower than that of patients with malignant tumors (43.0 vs. 54.4 years) $(p<0.05)$ (Figure 1$)$.

There were 8 metastatic lesions among malignant tumors, from: large intestine $(n=5)$, kidney $(n=2)$ and prostate $(n=1)$ and 2 primary lesions - teratocarcinoma $(n=1)$ and carcinoid $(n=1)$.

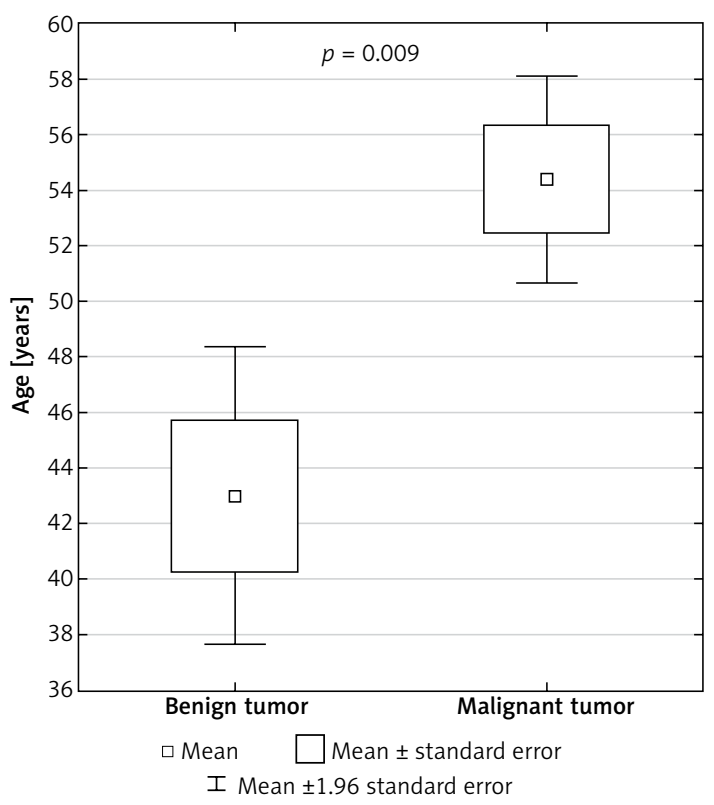

Figure 1. Average age of patients with benign and malignant presacral tumors 
The benign tumors included: cysts $(n=5)$, fibromas $(n=3)$, teratoma $(n=2)$, hamartoma $(n=1)$, neurogenic tumor $(n=1)$, lipoma $(n=1)$ and tumors of inflammatory etiology $(n=6)$.

Thirty-eight percent of cases were asymptomatic. Statistical analysis did not reveal a correlation between presence of complaints related to a presacral tumor and histology of the tumor. Chronic pain of the sacral region was the most common symptom $(n=13,45 \%)$. Other reported symptoms included constipation ( $n=5,17 \%)$, fever $(n=4,14 \%)$, vomiting $(n=4,14 \%)$, pain upon defecation $(n=3,10 \%)$ and loss of body weight $(n=3,10 \%)$ and benign lesions with respect to presenting symptoms (Table I).

Statistical analysis did not reveal a correlation between histology of the presacral tumor and average time from the first symptoms to initiation of surgical treatment (11 months).

All tumors were evaluated with preoperative imaging studies. Tumors in the presacral space were evaluated based on TRUS imaging in $83 \%$ of patients $(n=24)$, CT in $66 \%$ of patients $(n=19)$ and MRI in $17 \%$ of patients $(n=5)$. Seventeen percent of patients $(n=5)$ required TRUS, CT and MRI to provide detailed assessment of an advanced lesion.

Imaging studies determined the location of the tumors in relation to the S3 vertebra to enable selection of the surgical approach. In $52 \%$ of cases $(n=15)$, the upper margin of the tumor extended beyond the S3 vertebra, and in $48 \%$ of cases $(n=14)$ the whole tumor was below this level.

A transsacral approach was used in $51 \%$ of patients $(n=15)$, abdominal laparotomy in $41 \%$ of patients $(n=12)$ and a mixed approach in $7 \%$ $(n=2)$. The coccygeal bone was resected along with the tumor in $10 \%$ of patients $(n=3)$ due to a high degree of tumor progression. Partial rectal resection was required in $21 \%$ of cases $(n=6)$ due to malignant infiltration in 5 patients and due to intraoperative injury of the rectum in 1 case.

Cure was achieved in $72 \%$ of patients $(n=21)$, including in $67 \%$ after a first surgical procedure $(n=14)$. Thirty-three percent of patients $(n=7)$ required reoperation due to impaired drainage and development of hematoma $(n=4)$, recurrent malignancy $(n=2)$ and incomplete resection of the lesion $(n=1)$. In $28 \%$ of patients $(n=8)$ the tumor was assessed as unresectable during the procedure due to extensive infiltration of the surrounding structures of the pelvis minor that was not demonstrated by imaging studies.

Early postoperative complications up to 30 days after the procedure were found in $21 \%$ of patients $(n=6)$ : bleeding $(n=2)$, wound infection $(n=2)$, urethral injury $(n=1)$ and injury of the rectal wall $(n=1)$. Long-term complications were reported in $34 \%$ of cases $(n=10)$, and low back pain was the most common of them $(n=4)$. No correlation was found between the histology and incidence and nature of early complications.

The average patient follow-up was 4 years, and $7 \%$ of patients died during this period $(n=2)$. Both these deaths were caused by a malignant presacral tumor - 1 case of teratocarcinoma and 1 case of a metastatic lesion. In the teratocarcinoma case, the surgical resection of the primary lesion was a palliative procedure, while in the

Table I. Symptoms reported in patients with presacral tumors

\begin{tabular}{|c|c|c|c|c|c|c|}
\hline \multirow[t]{2}{*}{ Symptoms } & \multicolumn{2}{|c|}{ Benign tumors $(n=19)$} & \multicolumn{2}{|c|}{ Malignant tumors $(n=10)$} & \multicolumn{2}{|c|}{ Total $(n=29)$} \\
\hline & $n$ & $\%$ & $n$ & $\%$ & $n$ & $\%$ \\
\hline No symptoms & 6 & 32 & 5 & 50 & 11 & 38 \\
\hline Sacro-perineal pain & 9 & 47 & 4 & 40 & 13 & 45 \\
\hline Constipation & 3 & 16 & 2 & 20 & 5 & 17 \\
\hline Fever & 3 & 16 & 1 & 10 & 4 & 14 \\
\hline Vomiting & 3 & 16 & 1 & 10 & 4 & 14 \\
\hline Loss of body weight & 3 & 16 & 0 & 0 & 3 & 10 \\
\hline Painful defecation & 3 & 16 & 0 & 0 & 3 & 10 \\
\hline Dysuria & 1 & 5 & 1 & 10 & 2 & 7 \\
\hline Pencil thin stools & 1 & 5 & 0 & 0 & 1 & 3 \\
\hline Bloating & 1 & 5 & 0 & 0 & 1 & 3 \\
\hline Stool incontinence & 1 & 5 & 0 & 0 & 1 & 3 \\
\hline Neurosis & 1 & 5 & 0 & 0 & 1 & 3 \\
\hline Blood in the stool & 1 & 5 & 0 & 0 & 1 & 3 \\
\hline
\end{tabular}


case of the metastatic lesion, the malignant process became metastatic, resulting in death of the patient.

Local recurrence was found in $11 \%$ of patients with a benign lesion and in $40 \%$ of patients with a malignant lesion. The neoplastic process recurred more often in malignant than benign cases $(p<0.05)$. Recurrences of benign tumors were caused by lack of complete resection.

In $7 \%$ of patients $(n=2)$ gas and stool incontinence occurred after resection of a presacral tumor. In both these cases the tumors were malignant. However, no significant correlation between histology or surgical approach and anorectal disorders was found.

\section{Discussion}

The presacral region is a potential space located in the pelvis minor. The rectum is its anterior wall, the presacral fascia covering the sacral bone and coccygeal bone is its posterior wall, the peritoneal recess is its superior border, while the lavatory ani muscle is its inferior wall; its lateral borders are delineated by the iliac vessels and ureters. This space includes multiple branches of the sacral plexus, the pelvic visceral nerves, hypogastric nerves and autonomic innervations from the superior and inferior hypogastric plexus. Iliosacral, middle rectal, medium sacral vessels and plexuses of the presacral vein create a rich vascular network here. The whole presacral region is filled by reticular tissue and adipose tissue along with a network of lymphatic vessels. The rich vascular supply and presence of lymph nodes favor location of malignant metastases there. During embryogenesis, this space includes the notochord, large intestine, and posterior intestine, which involutes with time. Due to such diverse embryogenesis, the presacral region can be a site of variable neoplastic lesions, originating from all three embryonic germ layers [1, 5, 7].

Presacral tumors are a heterogeneous group of lesions with diverse histology. The literature classifies them into benign or malignant and congenital or acquired lesions. Further subclassification is based on the tumor histology [8]. According to the literature, the most common presacral tumor is the congenital benign teratoma. However, our study did not support this - as many as $93.1 \%$ of lesions were acquired and only 2 tumors were congenital, and they were indeed teratomas. This discrepancy may result from a small sample size and age range excluding patients below 18 years of age, in whom congenital lesions may have already been diagnosed and radically cured at earlier ages [8].

With regard to histology, benign tumors predominated in our study (65.5\%), including mainly inflammatory lesions and cysts. Metastases accounted for most of the malignant tumors; again according to literature reports, chordoma is the most common tumor in this setting. In our study sample, benign tumors predominated in women. Malignant tumors accounted for $34.5 \%$ of presacral tumors and predominated in men (80\%) (Table II) [1, 9-15]. These results are supported by literature data indicating that benign tumors are more common in women, malignant ones in men. Benign lesions are more common in younger subjects. This was also true in our study, where the average age of patients with a benign tumor was 43 years, and with a malignant one 54.4 years. Comparing these results with those of other authors may be biased because many researchers exclude from their study groups the pediatric population [9-15].

Presacral tumors are usually diagnosed late due to a lack of specific clinical signs and symptoms or their asymptomatic nature. Approximately 26$50 \%$ of patients, in the literature, are asymptotic, which is mirrored by our study (38\% of patients did not experience any clinical symptoms). Pain accompanying presacral tumors is dull, poorly localized and radiating. Presence of symptoms is believed to indicate complications of presacral tumors, resulting from infiltration of adjacent nerves or vessels. Infiltration of the sacral plexus causes pain of the lower extremities and buttocks. Presacral tumors may be one of the causes of lower back pain. Patients often complain of piercing sacral or perineal pain and gas or stool incontinence $[1,4,7,14]$. Pain in the sacral or perineal region may also be caused by ongoing inflammation in the pelvis minor. Uncharacteristic chronic sacro-perineal pain was the most common symptom in our study (occurring in $45 \%$ of patients). Various degrees of urine, stool and gas incontinence may result from infiltration or mechanical compression of pelvic nerves; stool incontinence was observed in 3\% of the study population. Expanding presacral tumors may cause mechanical obstruction of the pelvic outlet and obstruct defecation. Seventeen percent of patients in the study group experienced constipation, 10\% reported painful defecation and $3 \%$ reported pencil thin stools. The uncharacteristic clinical presentation of lesions in this region may result in establishment of incorrect diagnosis: pilonidal sinus, fistula or perianal abscess. Therefore, diagnostic workup of presacral tumors should be based on a differential diagnosis that includes the above-mentioned disorders [1, 9-15].

Presacral tumors are usually accidental findings of digital rectal examination or vaginal examination conducted for unrelated reasons. This is due to the fact that they are indolent and asymptomatic. Digital rectal examination is conducted in all patients, and our study indicates that its sensitivity approaches $62 \%$ [14]. Due to the lack 


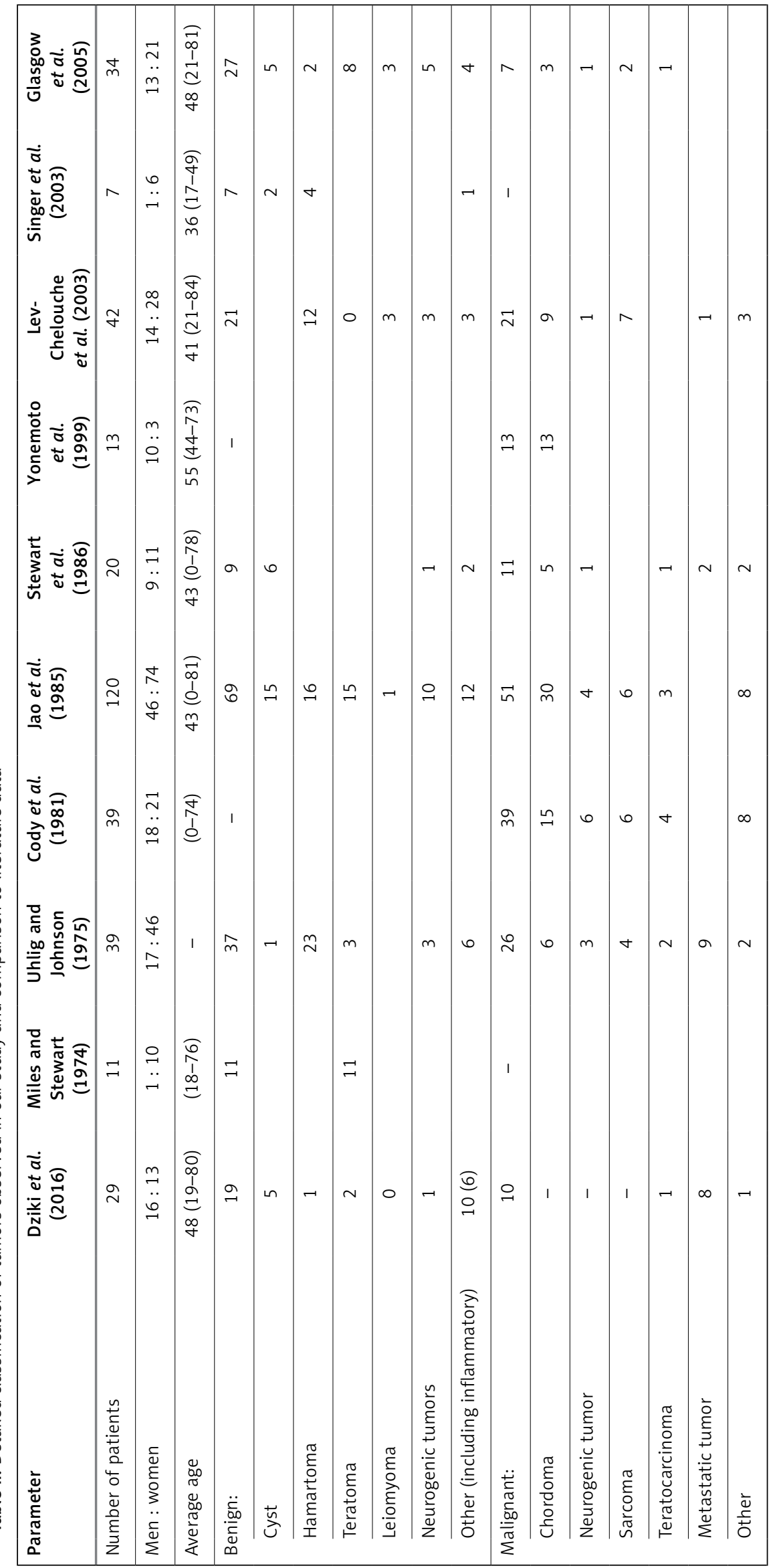


of characteristic signs and symptoms, diagnosis of a presacral tumor may be based on a detailed physical examination and interview, including detailed neurological examination. Rectoscopy to exclude colorectal pathologies should be the first additional test to be performed $[14,16]$.

Detailed imaging of the tumor and adjacent tissues should be an important part of the diagnostic management of presacral tumors. The CT and MRI seem to be the gold standard, and the best outcomes are provided by a combination of these two modalities. CT best visualizes the skeletal parts, the nature of the tumor - solid or cystic and infiltration of adjacent anatomical structures. On the other hand, MRI visualizes soft tissues and detailed spatial relations between the structures of the pelvis minor, which is very important for planning a surgical procedure $[8,14,17,18]$.

Preoperative biopsy is of utmost importance for determination of further therapeutic management, as with any tumor [2]. In presacral tumors such a procedure is particularly problematic due to difficult access to the lesion. However, the results of this investigation determine further therapeutic management. It is especially important in Ewing's sarcomas and chordomas, since in such cases neoadjuvant chemotherapy should be initiated. Based on the experience of various centers, Dozois et al. prepared recommendations on preoperative biopsy [2]. Biopsy should be performed by a radiologist, using a transperineal or transsacral approach with concurrent resection of the lymph node package. Before the procedure each patient should undergo blood clotting tests to reduce the risk of bleeding and biopsy specimen contamination.

Neoadjuvant chemotherapy is an important therapeutic component for selected types of presacral tumors such as Ewing's sarcoma, osteosarcoma or chordoma. An imatinib (tyrosine kinase inhibitor) treatment cycle is recommended in the therapy of these tumors. Transcatheter arterial embolization (TAE) is recommended for chordomas to increase the effectiveness of resection [19].

The role of radiotherapy in the treatment of presacral tumors is not clearly documented and is not associated with a good therapeutic effect. Carbon ion radiotherapy (CIRT) can be used in the treatment of chordomas. Positron emission tomography (PET) is usually performed to monitor therapeutic effectiveness in patients. Preoperative treatment is used to reduce the size of the tumor and improve the 5-year survival rate [2].

After detailed diagnostic workup and possible neoadjuvant therapy, each patient with a presacral tumor is prepared for the surgical procedure [19]. A surgical team should be provided with a complete set of imaging studies to determine the appropriate approach and resection margin. Preferably in all patients nutritional status should be assessed before surgery and supplemented with total parenteral nutrition (TPN) or enteral nutrition when indicated. Insertion of an inferior vena cava filter is recommended to reduce cardiovascular complications during the procedure. $\mathrm{Pa}$ tients who underwent radiotherapy should undergo temporary catheterization. In presacral tumors a team of anesthesiologists should be prepared for possible intraoperative blood transfusion due to the high risk of intraoperative bleeding. Each patient should receive a prophylactic dose of an antibiotic $48 \mathrm{~h}$ before the procedure, and an enema should be used to cleanse the bowels [13].

Selection of the surgical procedure depends on multiple factors. The following factors should be considered in the qualification process: tumor size, its precise location in relation to adjacent anatomical structures, degree of infiltration of adjacent tissues and degree of vascularization. Special attention should be paid to its relation to the $\mathrm{S3}$ vertebra, location relative to the rectum and adjacent vascular plexuses and nerves [13, 20-24].

Currently several methods of surgical approach to the presacral region are known and used. They include transsacral, abdominal, mixed abdomino-sacral, transrectal (transsphincter) and transvaginal approaches. Despite the fact that presacral tumors belong to the area of open surgery, there are also reports documenting laparoscopic techniques used for such treatment [13, 20-24]. Transsacral, abdominal, and mixed abdomino-sacral procedures are most common. Small lesions that do not exceed $1 \mathrm{~cm}$, located below the third sacral vertebra (S3), allow for transsacral resection. Lesions with a diameter larger than $1 \mathrm{~cm}$ or located above S3 undergo resection using an abdominal or mixed approach.

Each procedure requires general anesthesia [20-24].

A transsacral or posterior approach involves accessing the presacral space through a vertical incision, approximately $8-10 \mathrm{~cm}$ in length, at the S3 vertebral level. Sometimes the sacral or coccygeal bone needs to be resected to enable radical resection [20-24]. Intraoperative assessment of the tumor location and degree of involvement of adjacent tissues in the presacral space are important aspects of this procedure. Special caution in tumor dissection is advised due to the rich and variable blood supply of this region. The advantage of the procedure with a transsacral approach is shorter postoperative recovery. The transsacral approach, without the abdominal approach, is the most commonly selected method [20-24]. This is also supported by our study: $51 \%$ of patients underwent a transsacral procedure.

The abdominal, anterior approach (laparotomy) is used for the treatment of larger tumors, with the margin located above the S3 vertebra level. Resection from the abdominal approach involves 
accessing the presacral space through a midline incision in the lithotomy position. This procedure requires careful dissection of vessels and the mesorectal space. Damage of sacral artery branches may result in intraoperative bleeding that is difficult to manage [25-27]. Abdominal resection is commonly used, which is supported by our study: this was the method of choice in $41 \%$ of patients diagnosed with a presacral tumor.

A mixed, abdomino-sacral approach, combining both the above-mentioned techniques, is also commonly used. It is preferred for the treatment of large tumors located above the S3 vertebra, with marked involvement of surrounding tissues. Seven percent of our patients underwent this procedure [25-27].

Very small and low-lying tumors may be resected using a transsphincter or transvaginal approach; however, these methods are used uncommonly [20, 22, 25-27].

Irrespective of selection of the method, surgical treatment aims at complete resection of the tumor with a large margin of healthy tissues to obtain the best possible treatment outcomes. Complete tumor resection increases the chance of cure and reduces the recurrence rate [25-27].

Postoperative radiotherapy is not a standard management of presacral tumors. Treatment of some malignant tumors may be supplemented by a radiotherapy cycle, but its efficacy has not been definitely proven [28].

If the presacral tumor is a cyst or an abscess, the management of choice is to remove it after its drainage using a percutaneous drainage set [25].

Irrespective of selection of the surgical approach, the procedure is associated with high in tra- and perioperative risk of complications. The most common complications include hemorrhage, rectal injury, injury of sacral plexus nerves or injury of the urethra [28-31]. Complications of a similar nature were found in $26 \%$ of patients in our study. Bleeding, wound infection, and injury of the urethra or rectum were the most common. Bleeding from presacral venous plexuses is the most common cause of intraoperative deaths in patients undergoing surgical treatment for a tumor located in this space. According to the literature, preoperative embolization of blood vessels supplying the tumor and intraoperative pressure control reduce the bleeding risk [25, 28-31]. Lack of tumor recurrence is an important aspect of effectiveness of a surgical procedure. Appropriate oncological cleanness associated with resection of the tumor with an adequate margin of healthy tissue minimizes the risk of local recurrence. Neoplasm recurrence occurs more often with malignant tumors. Chondroma has the highest recurrence rate [29]. According to various studies, presacral tumors recur in $0-15 \%$ of cases [20-31]. If a benign tumor is completely resected, we can assume that the local recurrence rate is $0 \%$. Such outcomes are supported by large studies performed by Glasgow et al., demonstrating that the recurrence rate in 22-month follow-up was 0\% [14]. In our study the local recurrence rate was $11 \%$ for patients with benign tumors and $40 \%$ with malignant tumors. Recurrences were caused by lack of complete tumor resection in cases of benign tumors.

Patient survival is an important aspect of effectiveness of therapy. According to studies conducted by Mayo Clinic, 5-year survival after the surgical therapy is 75\% [1]. Cody, Macrove, and Quan reported 69\% 5-year survival and 50\% 10year survival rates in patients who had undergone surgical treatment for presacral tumors [10, 32]. Spanish studies conducted in Valencia documented a $90 \%$ survival rate over 3.5 years of follow-up [29]. All the above figures refer to malignant tumors. In benign tumors, complete tumor resection does not affect the patient survival. Two patients died during 4 years of follow-up in our study. In the first case of teratocarcinoma, resection of the primary lesions was a palliative procedure, while in the second case, after resection of a metastatic lesion the malignancy became metastatic, resulting in the patient's death.

In conclusion, presacral tumors are a rare condition with an unspecific clinical presentation. Lesions with such location are associated with diagnostic difficulties and are often diagnosed late. Assessment of tumors with imaging studies is a key component for selection of the technique of surgical resection that is the principal therapy for these lesions. Presacral tumors are more common in men and more commonly are malignant tumors in this group. Benign tumors are more common in women. The success rate of surgical treatment is $72 \%$ and selection of the surgical approach does not affect the final treatment outcome. Recurrences are more common in malignant tumors.

\section{Conflict of interest}

The authors declare no conflict of interest.

\section{References}

1. Jao SW, Beart RW, Spencer RJ, Reiman HM, Ilstrup DM. Retrorectal tumors. Mayo Clinic experience, 1960-1979. Dis Colon Rectum 1985; 28: 644-52.

2. Dozois EJ, Jacofsky DJ, Dozois RR. Presacral tumors. In: The ASCRS textbook of colon and rectal surgery. Wolff BG, Fleshman JW, Beck DE, Pemberton JH, Wexner SD (eds). Springer Science Business Media, New York 2007; 501-14.

3. Young-Fadock TM, Dozois RR. Tumores retrorrectales. In: Shackelford. Cirugía del aparato digestivo. 5th ed. Zuidema GD, Yeo CJ (eds.). Buenos Aires: Médica Panamericana 2005; 534-43. 
4. Scullion DA, Zwirewich CV, McGregor G. Retrorectal cystic hamartoma: diagnosis using endorectal ultrasound Clin Radiol 1999; 54: 338-9.

5. Hobson KG, Ghaemmaghami V, Roe JP, Goodnight J, Khatri VP. Tumors of the retrorectal space. Dis Colon Rectum 2005; 48: 1964-74.

6. Lee RA, Symmonds RE. Presacral tumors in the female: clinical presentation, surgical management, and results. Obstet Gynecol 1998; 71: 216-21.

7. López M, Vilallonga R, Espin E, Sánchez JL, Lozoya, Armengol M. Lesiones retrorrectales en adultos. Experiencia en 5 casos. Cir Esp 2006; 80: 334-6.

8. Lcv-Chclouche D, Gutman M, Goldman G, el al. Presacral tumors: a practical classification and treatment of a unique and heterogeneous group of diseases. Surgery 2003; 133: 473-78.

9. Uhilig BE, Johnson RL. Presacral tumors and cysts in adults. Dis Colon Rectum 1975; 18: 581-96.

10. Cody HS, Macrove RC, Quan SH. Malignant retrorectal tumors: 28 years' experience at Memorial Sloan-Kettering Cancer Center. Dis Colon Rectum 1981; 24: 501-6.

11. Stewart RJ, Humphreys WG, Parks TG. The presentation and management of presacral tumors. Br J Surg 1986; 73: 153-5.

12. Yonemoto T, Tatezaki SH, Takenouchi T, Ishki T, Satoh T, Moriya $\mathrm{H}$. The surgical management of sacrococcygeal chordoma. Cancer 1999; 85: 878-83.

13. Singer MA, Cintron JR, Martz JE, Schoetz DJ, Abcarian H. Retrorectal cyst: a rare tumor frequently misdiagnosed. J Am Coll Surg 2003; 196: 880-6.

14. Glasgow SC, Birnbaum EH, Lowney JK, et al. Retrorectal tumors: a diagnostic and therapeutic challenge. Dis CoIon Rectum 2005; 48: 1581-7.

15. Miles RM, Stewart GS Jr. Sacrococcygeal teratomas in adult. Ann Surg 1974; 179: 676-83.

16. Ghosh J, Eglinton T, Frizelle FA, Waston AJ. Presacral tumors in adults. Surgeon 2007; 5: 31-8.

17. Testini M, Catalano G, Macarini L, Paccione F. Diagnosis and surgical treatment of retroperitoneal tumors. Int Surg 1996; 81: 88-93.

18. Dahan H, Arrive L, Wendum D, Docoii IP, Djoubri H, Tubiana JM. Retrorectal developmental cysts in adults: clinical and radiologic-histopathologic review, differentia diagnosis, and treatment. Radiographics 2001; 21: 575-84.

19. Hassan I, Wietfeldt ED. Presacral tumors: diagnosis and management. Clin Colon Rectal Surg 2009; 22: 84-93.

20. Dozois RD, Chiu LK. Retrorectal tumors. In: Surgery of the Colon and Rectum. Nicholls RJ, Dozois RR (eds.) Churchill Livingstone, New York 1997; 533-45.

21. Pidala MJ, Eisenstat TE, Rtibin RJ, Salvati EP. Presaeral cysts: transrectal excision in select patients. Am Surg 1999; 65: 112-5.

22. Abel ME, Nelson R, Prasad ML, Pearl RK, Orsay CP, Abcarian $\mathrm{H}$. Parasacrococcygeal approach for the resection of retrorectal development cysts. Dis Colon Rectum 1985; 28: 855-8.

23. Locaiio SA, Eng K, Ranson JH. Abdominosacral approach for retrorectal tumors. Ann Surg 1980; 191: 555-60.

24. Braley SC, Schneider PD, Bold RJ, Goodnight JE Jr, Khatri VP. Controlled tamponade of severe presacral venous hemorrhage use of a breast implant sizer. Dis Colon Retum 2002; 45: 140-2.

25. Woodfield JC, Chalmers AG, Phillips N, Sagar M. Algorithms for the surgical management of retrorectal tu mours. Br J Surg 2008; 95: 214-21.

26. Jackson RJ, Gokaslan ZL. Spinal-pelvic fixation in patients with lumbosacral neoplasms. J Neurosurg 2000; 92 Suppl. 1: 61-70.
27. MacCarty CS, Waugh JM, Coventry MB. Surgical treatment of sacral and presacral tumors other than sacrococcygeal chordoma. J Neurosurg 1965; 22: 458-64.

28. Fuller DB, Bloom JG. Radiotherapy for chordoma. Int J Radiat Oncol Biol Phys 1998; 15: 331-9.

29. Canelles E, Qoiq JV, Cantos M, et al. Presacral tumors: analysis of 20 surgically treated patients. Cir Esp 2009; 85: 371-7.

30. Stener B. Resection of the sacrum for tumors. Chir Organi Mov 1990; 75 (Suppl. 1): 108-10.

31. Todd LT Jr, Yaszemski MJ, Currier BL, Fuchs B, Kim CW, Sim FH. Bowel and bladder function after major sacra resection. Clin Orthop Relat Res 2002; 397: 36-9.

32. Longo JM, Bilboa JI, deVilla VH, et al. CT-guided paracoccygeal drainage of pelvic abscesses. J Comput Assist Tomogr 1993; 17: 909-14. 